\title{
Analgesic and Sedative Effects of Blue LED Light in Combination with Infrared LED Irradiation
}

\author{
Sergiy A. Gulyar ${ }^{1,2}$ and Zynaida A. Tamarova ${ }^{1}$ \\ 1. Department of General Physiology of Nervous System, Bogomoletz Institute of Physiology National Academy of Sciences of \\ Ukraine, Kiev 401601, Ukraine
}

2. International Medical Innovation Center Zepter, Kiev 02152, Ukraine

\begin{abstract}
INTRODUCTION: Our previous studies have shown that polarized red+infrared LED light causes analgesic effect. The aim of this study was to test whether the blue LED light has such effect. We compared the analgesic efficacy of continuous blue light without infrared component and in combination with infrared irradiation. We also determined the dependence of responses on frequency of pulsation of blue+infrared (blue+IR) LED irradiation. METHODS: We used the model of experimentally induced chemical inflammation (formalin test). The source of the light emission was MEDOLIGHT-BluDoc device. In animals (white mice males), we recorded the duration of pain (licking the affected limb) and non-painful (sleeping, washing, running, food) behavioral reactions with placebo (control) and after the 10-minute light application on the locus of pain. The influence of continuous (blue or blue+IR) and pulsed (frequency of pulsations $10 \mathrm{~Hz}, 600 \mathrm{~Hz}, 3000 \mathrm{~Hz}$ and $8000 \mathrm{~Hz}$ ) (blue+IR) light was determined. RESULTS: After the application of blue LED light without infrared component or with infrared radiation duration of pain response decreased to $317.3 \mathrm{~s}$ and $282.3 \mathrm{~s}$ against $566.2 \mathrm{~s}$ in the control group. Analgesia was $43.6 \%$ and 50.1\%. Pulsed blue+IR LED light (all the tested ranges) also significantly reduced pain. Analgesia was maximal at a frequency of $8000 \mathrm{~Hz}(57.6 \%)$ and minimal at a frequency of $10 \mathrm{~Hz}(38.3 \%)$. Duration of sleep in experimental groups was 2.3-3.8 times longer than in the control group, the effect was most noticeable at frequency of $10 \mathrm{~Hz}$. Conclusion: It was found that the blue and blue+IR LED light MEDOLIGHT-BluDoc device (both continuous and pulsing mode) when applied to a painful inflammation area possess a significant analgesic and sedative effect.
\end{abstract}

Key words: Formalin-induced pain, analgesia, blue LED light, infrared LED irradiation, continuous light, pulsed light, blue+infrared LED light.

\section{Introduction}

Blue range of the visible spectrum of light occupies a special place in regulation of many photo dependent processes in plants and mammals. Converting of light energy into chemical (photosynthesis) is the most important process in plant development. Regulation of morphogenetic processes in animals and humans is also largely provided by shortwave light with electromagnetic radiation in the range of $420-480 \mathrm{~nm}$. It has a higher penetrating ability in aqueous medium, down to $250 \mathrm{~m}$ as compared with $30 \mathrm{~m}$ for ultraviolet and $15 \mathrm{~m}$, for red light. Due to this and taking into account the fact that the body tissue saturated with

Corresponding author: Sergiy A. Gulyar, M.D., Ph.D., DSci, professor, research field: environmental physiology and medicine. water in different ways, even deeply located tissue molecules absorb additional quanta of energy. There increases the activity of metabolic processes, of many photosensitive enzymes, flavins, cytochromes, carotenoids and others. As a result, at the organ level, we observe increase of microcirculation, improvement of rheological properties of blood (clotting and blood viscosity, disaggregation of erythrocytes), destruction of bilirubin excess, atherogenic lipids and other photo dependent processes [1].

Photochemical mechanism of light depended phosphorylation consist in the following: at lightening by blue light there occurs excitation of mitochondria flavoproteins. Transfer of electrons leads to energy elimination which can be used for phosphorylation [2]. In animal cells, there are many structures that absorb 
optical radiation at wavelengths of 430-475 $\mathrm{nm}$. To them belong flavins, which are coenzymes of essential enzymes (flavin nucleotides): NADH dehydrogenase, succinate dehydrogenase, acyl-CoA dehydrogenase, oxidase, D-amino acids, and glucose oxidase. The final electron acceptor for flavin dehydrogenases is the cytochrome system. They all contain iron porphyrin groups, capable of absorbing light with $450 \mathrm{~nm}$ wavelength. Porphyrin structures that absorb blue light have also bilirubin $(460 \mathrm{~nm})$ and hemoglobin $(420 \mathrm{~nm})$, protoporphyrin and blood porphyrins (440 $\mathrm{nm})$. Electromagnetic waves of light range under consideration are also well absorbed by carotenoids such as carotene (440 nm and $447 \mathrm{~nm}$ ), neuro spores (416 nm, $440 \mathrm{~nm}$ and $470 \mathrm{~nm}$ ), heart homogenate carotenoids $(450 \mathrm{~nm})$. Riboflavin absorbs blue light. At the same time, cellular structures that absorb green, yellow or red light are practically absent, except catalase, which absorbs red light. It is assumed that the mechanism of action of optical radiation in the range of $420-480 \mathrm{~nm}$ is realized through aqua structure of biological tissues [3].

At the same time the body is sensitive to all wavelength range of visible light, there are revealed appropriate responses. It was found out in the process of study of radiation effect of halogen light, laser and LED sources [4-7]. However, quantum-wave nature of light radiation assumes that tissues must respond not only to its wavelength range, but also to their frequency. Most studies describe different directions of continuous light action (mixed white, red, orange, etc.). For intermittent (pulsed) light (PL), the data available in the literature are contradictory, which does not allow to highlight its significant differences from continuous. There is an opinion about the best therapeutic efficacy of pulsed mode that explains the deeper penetration of light [7]. It is therefore possible for practical problems in physiotherapy to use empirically selected frequencies of electromagnetic radiation in a very wide range [8].

Our previous studies have shown that red+IR polarized LED light significantly reduces the formalin-induced pain response $[9,10]$. It has been found that it is as effective and sometimes superior to red halogen light $[11,12]$. At equal exposure analgesia was $55.9 \%$ and $45.7 \%$, respectively.

Influence of the blue light on the pain response, we investigated previously only for halogen light (BIOPTRON device was equipped with a blue light filter). At the formalin test model in mice when polarized blue light was applied to the locus of pain, we registered significant analgesic effect $[11,12]$, but it was less $(31.5 \%)$ than in the case with red halogen light $[9,10]$.

The aim of this study was to obtain objective evidence of analgesic efficacy of blue LED light in continuous and pulsed modes and also in combination with IR irradiation.

We have set the task to study animals with hemogenic inflammatory locus (formalin test), how changes the one of the main indicators of inflammation-pain. We evaluated its intensity after continuous application of LED blue light without infrared component and with infrared component, and the blue+IR LED light with frequency interruption of $10 \mathrm{~Hz}, 600 \mathrm{~Hz}, 3000 \mathrm{~Hz}$ and $8000 \mathrm{~Hz}$ on inflamed skin. MEDOLIGHT-BluDoc (Zepter/BIOPTRON, Switzerland) served as the light source unit.

\section{Materials and Methods}

\subsection{Animals}

Studies were conducted on white laboratory mice with formalin-induced inflammatory area (formalin test). Formalin test is a classical model of hemogenic tonic pain $[13,14]$. It is widely used in many laboratories to check the analgesic effectiveness of pharmacological analgesic drugs and physical therapy techniques.

All experiments were performed in accordance with ethical guidelines of the International Association for the Study of Pain. We used 85 adult albino male mice weighing 28-32 g. Animals were kept at controlled 
temperature $\left(18-20^{\circ} \mathrm{C}\right)$ and 12-hour photoperiod. Access to food and water was free. No later than a day before the experiment, mice were seated in individual plastic cages $(36 \times 24 \times 16 \mathrm{~cm})$ and carried into the room where the studies were conducted to adapt. Animals were randomly divided into 7 groups: the experimental 6 (10 mice in each) and one control (comparative placebo) (15 mice). Each mouse was used only in one experiment and after the end of it was euthanized with a lethal dose of urethane. All experiments were performed at the same time of the daylight hours (10 to 13 hours) to lower the contribution of circadian effects on nociceptive sensitivity [15].

\subsection{Formalin Test}

Inflammation area was created by the subcutaneous injection of a 5\% solution of formalin into the dorsal surface of the foot of the left hind limb. The injection site showed redness and swelling, which remained for several hours. One of the main indicators of the inflammatory process is pain syndrome. Licking the injected limb - a typical behavioral response to pain. It is known that formalin-induced pain response has two phases $[13,14]$. The early phase (acute pain, pain caused by irritation of skin receptors) begins immediately after formalin injection and lasts up to 10 minutes. Late phase (tonic pain, which is a consequence of the inflammatory process) starts after 10 minutes and lasts for more than an hour. Since in our experiments, immediately after formalin solution injection into a paw we made 10minute application of light on the locus of inflammation, we could not see the early phase of the pain response. Further we will talk only about the late phase - tonic pain.

During the formalin injection and a subsequent light application mouse was in a narrow plastic chamber. The left hind limb was put through a hole outside the chamber and gently hand-held by the experimenter. At the end of a session of light therapy or imitation of such a session (the control group) mouse, was released in his cage and we recorded the duration of pain response (licking the affected limb) and non-painful reactions (sleeping, washing, running, eating) over the successive 10-minute periods of time and for the whole observation period (60 min).

\subsection{Applications of Blue LED or Blue+Infrared LED Light}

Immediately after the formalin injection, experimental animals received application of LED light on the locus of pain using MEDOLIGHT-BluDoc

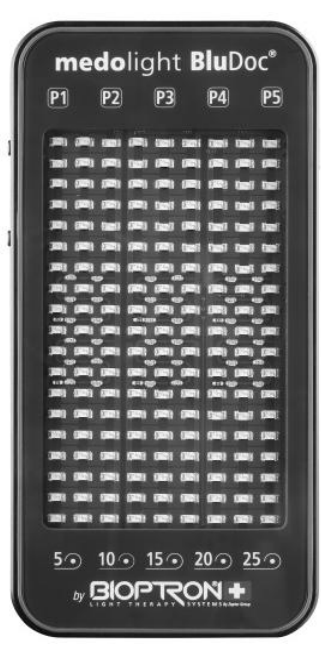

(A)

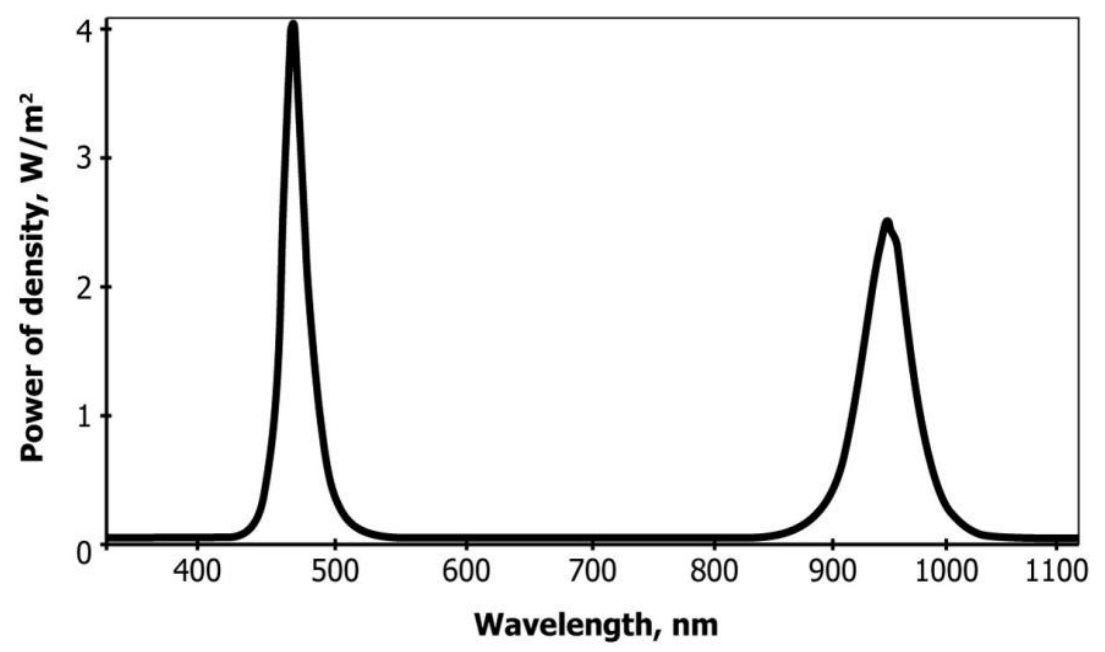

(B)

Fig. 1 MEDOLIGHT-BluDoc device and its radiation wavelength range.

(A) MEDOLIGHT-BluDoc device, P1-P5 — modes $(0 \mathrm{~Hz}, 10 \mathrm{~Hz}, 600 \mathrm{~Hz}, 3000 \mathrm{~Hz}$ and $8000 \mathrm{~Hz}), 5-25$ - exposure value (min);

(B) blue $(470 \mathrm{~nm})$ and infrared $(940 \mathrm{~nm})$ LEDs wavelength peaks measured by BLUE-Wave Spectrometer VIS-25. Horizontally-the wavelength in nanometers, vertical-radiation power of density in $\mathrm{W} / \mathrm{m}^{2}$. 
device. Light power density at a distance of $0 \mathrm{~cm}$ was $10.15 \mathrm{~mW} / \mathrm{cm}^{2}$, and at a distance of $1 \mathrm{~cm}, 8.2 \mathrm{~mW} / \mathrm{cm}^{2}$. Wavelengths were 470 (467-477) nm (Light Emitting Diode with InGaN HL-PC-3216H203BC, Honglitronic) and $940 \pm 50 \mathrm{~nm}$ (Infrared Emitting Diode with Gallium Arsenide KP-1608F3C, Kingbright), radiation energy $2.75 \mathrm{~J} / \mathrm{cm}^{2}$. Due to small size of the pain locus we applied light emanation from two neighboring LEDs. The other LEDs were closed black impenetrable film. We used irradiation of 2 blue or blue+infrared (IR) LEDs. The distance from the surface of the skin to the light source was 1-3 mm, exposure $10 \mathrm{~min}$.

A total of 6 experimental series: in two were applied blue continuous light (CL) or blue+IR continuous irradiation (CL), in four other series we used blue or blue+IR PL (10 Hz, $600 \mathrm{~Hz}, 3000 \mathrm{~Hz}$ or $8000 \mathrm{~Hz}$ ).

\subsection{Statistical Analysis}

With the help of a special computer program, we calculated the duration of painful and non-painful behavioral responses for each successive 10 minutes, and for the entire period of observation (60 min). Data are presented as mean \pm SEM. To determine the statistical significance of the results we used Student t-test. Differences were considered statistically significant at $P<0.05$.

\section{Results}

\subsection{Effect of Continuous Blue LED Light on Formalin-Induced Pain}

We compared pain behavior changes of animals under the influence of blue CL without IR component and with IR component on inflammation locus. Results obtained in two experimental groups of animals in comparison to similar reactions placebo group (control, without light application) are shown in Fig. 2.

In both groups, which used blue LED light throughout the observation period pain response to formalin was weaker than in the control group (without light application) (Fig. 2A). After 50-60 minutes, the pain in the experimental groups was practically absent, whereas in the control it continued to remain at a high level.
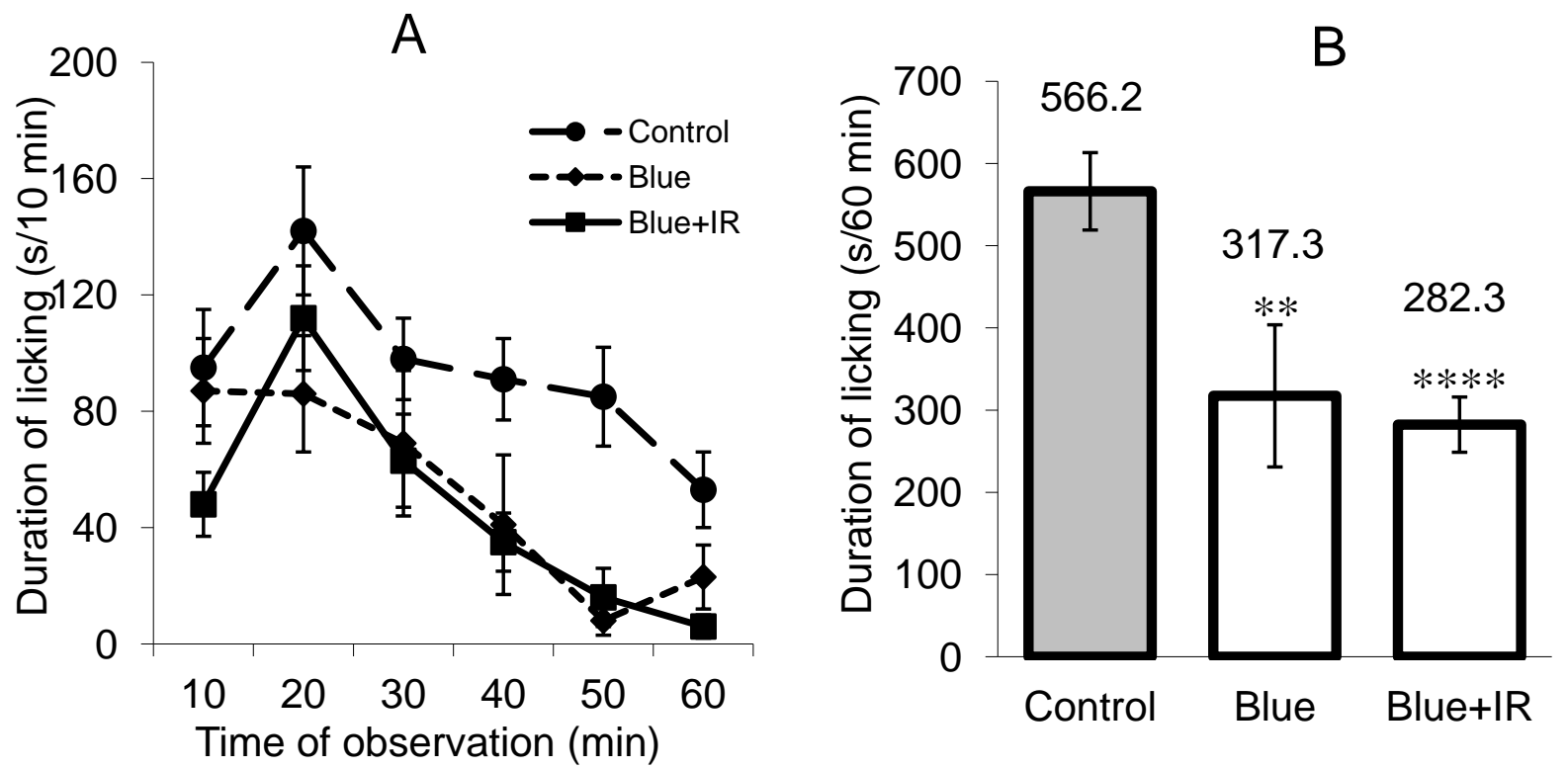

Fig. 2 Pain reactions after 10 minutes of application of blue CL without IR and with IR component on the locus of pain in comparison with the control.

(A) Dynamics of the reactions in the three groups. (B) Total duration of pain for 60 minutes of observation in the three groups. The bars represent mean \pm S.E.M. The numbers above the bars - the duration of the pain reaction in seconds.

Significance of differences with the control: **** $P<0.002 ; * * P<0.05$ 
Table 1 Average values of the duration (s and \% from control) of pain and non-painful behavioral reactions during 60 minutes in the control group (without application of light) and two experimental groups in which blue CL without IR component or with IR componentwere applied. Light acted on the pain locus within 10 minutes from the distance of 1-3 mm.

\begin{tabular}{llll}
\hline \multirow{2}{*}{ Behavioral responses } & Control (placebo) & Blue & Continuous LED light \\
\cline { 3 - 4 } Licking & $566.2 \pm 47.1$ & $317.3 \pm 86.5^{* * *}$ & Blue+Infrared \\
Sleeping & $100 \%$ & $56 \%$ & $282.3 \pm 33.6^{* * * * *}$ \\
& $386.3 \pm 79.3$ & $945.2 \pm 182.6^{* * *}$ & $896.8 \pm 165.1^{* * * *}$ \\
Washing & $100 \%$ & $244.7 \%$ & $232.2 \%$ \\
Running & $137.9 \pm 32.5$ & $191.3 \pm 25.1 *$ & $205.5 \pm 36.7^{*}$ \\
& $100 \%$ & $138.7 \%$ & $149 \%$ \\
Eating & $65.5 \pm 13$ & $113.6 \pm 85.7$ & $103.9 \pm 36.5^{*}$ \\
\hline
\end{tabular}

Significance of differences with the control group: $* * * * P<0.002 ; * * * P<0.005 ; * * P<0.05 ; * P<0.5$ (the rest is not significant).

When comparing totals (for 60 minutes of observation) of pain reactions in different groups, we observed statistically significant difference between each of the two experimental and control groups (Table 1, Fig. 2B).

After 10 minutes of application on the inflammatory locus by blue CL without IR component or with IR component, pain reaction duration was from $317.3 \mathrm{~s}$ to $282.3 \mathrm{~s}$ against $566.2 \mathrm{~s}$ in the control group. Significant differences with the control are very high. However, between the two experimental groups, as for duration of the pain the difference is not significant. Analgesia due to use of the blue LED light without IR component was $44 \%$ and with the IR component of $-50.1 \%$.

In both groups, in which we made application on the locus of pain by blue CL (both without IR and with IR component), we observed increase in duration of all the four non-painful behavioral reactions. All experimental animals as compared to control slept, washed, run, and ate longer (Table 1). These figures also indicate to the relief of pain. Statistically significant differences in the duration of non-painful reactions in two experimental groups were not revealed.

Of non-painful behavioral responses, the most significantly changed sleep. In the group, where the blue CL was used, total sleep duration increased 2.4 times, while the animals treated with the session of CL blue+IR -2.3 times compared with the control. There increased duration of eating periods (Table 1). These data allow us to conclude that the CL blue and blue+IR has not only analgesic, but also sedative effect.

\subsection{Effects of Pulsed Blue+IR Light at Frequencies of $10 \mathrm{~Hz}, 600 \mathrm{~Hz}, 3000 \mathrm{~Hz}$ or $8000 \mathrm{~Hz}$}

In experimentally induced models of somatic pain (formalin test), we studied non-painful and painful behavioral response after application of the PL blue+IR with frequencies of $10 \mathrm{~Hz}, 600 \mathrm{~Hz}, 3000 \mathrm{~Hz}$ and 8000 $\mathrm{Hz}$ on the inflammation locus.

In all experimental groups, we singled out significant difference in the dynamics of development of painful reactions compared to placebo data. In the groups, in which light applications were done, pain responses were weaker in all phases of observation (Fig. 3). After 40-60 minutes the pain almost subsided, whereas in the control group it continued to hold at a high level.

The total values of the pain duration within 60-minutes of observation period in all groups in which we applied PL, with a high degree of reliability differed from the control (Table 2, Fig. 4). We have seen that in all the experimental groups pain response was much weaker than in the control. The duration of pain was minimal at a frequency of pulsation of $8000 \mathrm{~Hz}(240 \mathrm{~s})$ and maximal at $10 \mathrm{~Hz}$ (to $349.2 \mathrm{~s}$ ). This amounted $42.4 \%$ and $61.7 \%$ of the same reaction in the control group $(566.2 \mathrm{~s}=100 \%)$.

Groups where the light was applied at a frequency of 
pulsation $3000 \mathrm{~Hz}$, and $600 \mathrm{~Hz}$, occupied intermediate values. Pain in these groups was $46.9 \%$ and $49.5 \%$ of control values. CL blue+IR as to its efficacy did not differ from the PL with a frequency of pulsation 600 $\mathrm{Hz}$. In this case, the mean value of pain duration was 282.3 s, i.e. $49.8 \%$ of the control value.

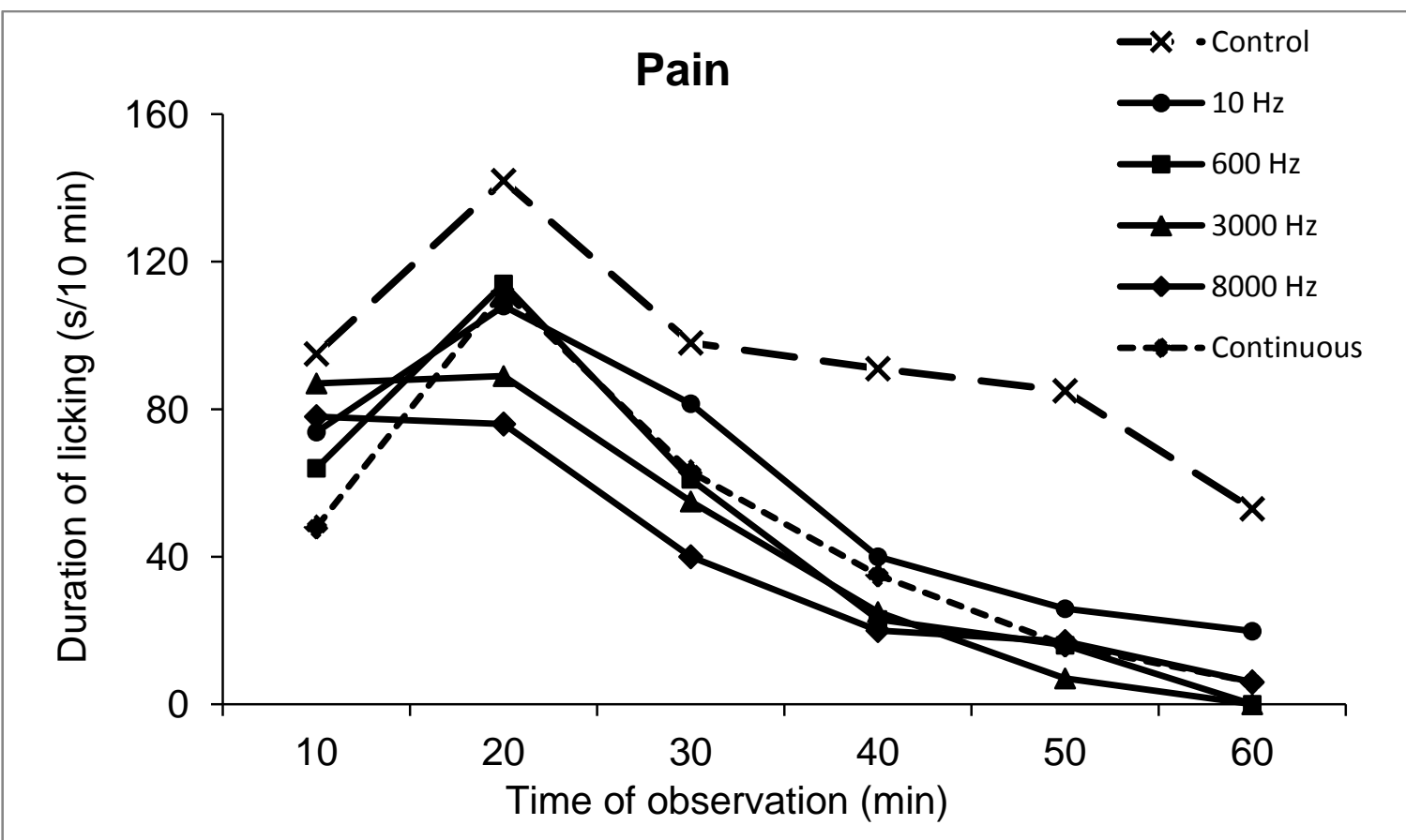

Fig. 3 Dynamics of pain response after application on the locus of pain PL blue+IR with pulsing frequencies of $10 \mathrm{~Hz}, 600 \mathrm{~Hz}$, $3000 \mathrm{~Hz}$ and $8000 \mathrm{~Hz}$.

For comparison it is given the painful reaction to the application of CL blue+IR and the response in the control group, where the light was not used.

Table 2 Mean duration (s and \% from control) of painful and non-painful behavioral reactions observed for 60 minutes of observations in the control group (without application of light) and after exposure to PL blue+IR (frequency interruption of 10 $\mathrm{Hz}, 600 \mathrm{~Hz}, 3000 \mathrm{~Hz}$ and $8000 \mathrm{~Hz}$ ). Light acted on the locus of pain during 10 minutes from a distance of 1-3 mm.

\begin{tabular}{llllll}
\hline \multirow{2}{*}{$\begin{array}{l}\text { Behavioral } \\
\text { responses }\end{array}$} & Control (placebo) & \multicolumn{3}{c}{ Continuous blue+infrared LED light } \\
\cline { 3 - 6 } & & $10 \mathrm{~Hz}$ & $600 \mathrm{~Hz}$ & $3000 \mathrm{~Hz}$ & $8000 \mathrm{~Hz}$ \\
\hline Licking & $566.2 \pm 47.1$ & $349.2 \pm 51.1 * * *$ & $280.4 \pm 54 * * *$ & $265.3 \pm 48.9 * * *$ & $240 \pm 45.3 * * * *$ \\
& $100 \%$ & $61.7 \%$ & $49.5 \%$ & $46.9 \%$ & $42.4 \%$ \\
Sleeping & $386.3 \pm 79.3$ & $1485.3 \pm 151.4 * * * *$ & $1090.8 \pm 186.1 * * *$ & $1324.6 \pm 181.3 * * *$ & $1287.1 \pm 204.9 * * *$ \\
& $100 \%$ & $384.5 \%$ & $282.4 \%$ & $342.9 \%$ & $333.2 \%$ \\
Washing & $137.9 \pm 32.5$ & $135.3 \pm 21.2$ & $132.5 \pm 21.3$ & $183.1 \pm 28.6 *$ & $175.9 \pm 23.1 *$ \\
& $100 \%$ & $98.1 \%$ & $96.1 \%$ & $132.7 \%$ & $127.5 \%$ \\
Running & $65.5 \pm 13$ & $72.3 \pm 20.9$ & $72.9 \pm 28.9$ & $33.4 \pm 9.1 *$ & $50.2 \pm 27.3$ \\
& $100 \%$ & $110.4 \%$ & $111.4 \%$ & $51 \%$ & $76.7 \%$ \\
Eating & $1.1 \pm 0.4$ & $0 \pm 0 * *$ & $33.6 \pm 21 *$ & $33.5 \pm 17.9 *$ & $39.6 \pm 27.3 *$ \\
\hline
\end{tabular}

Significance of differences with the control group: $* * * * P<0.001 ; * * * P<0.01 ; * * P<0.05 ; * P<0.5$ (the rest is not significant). 


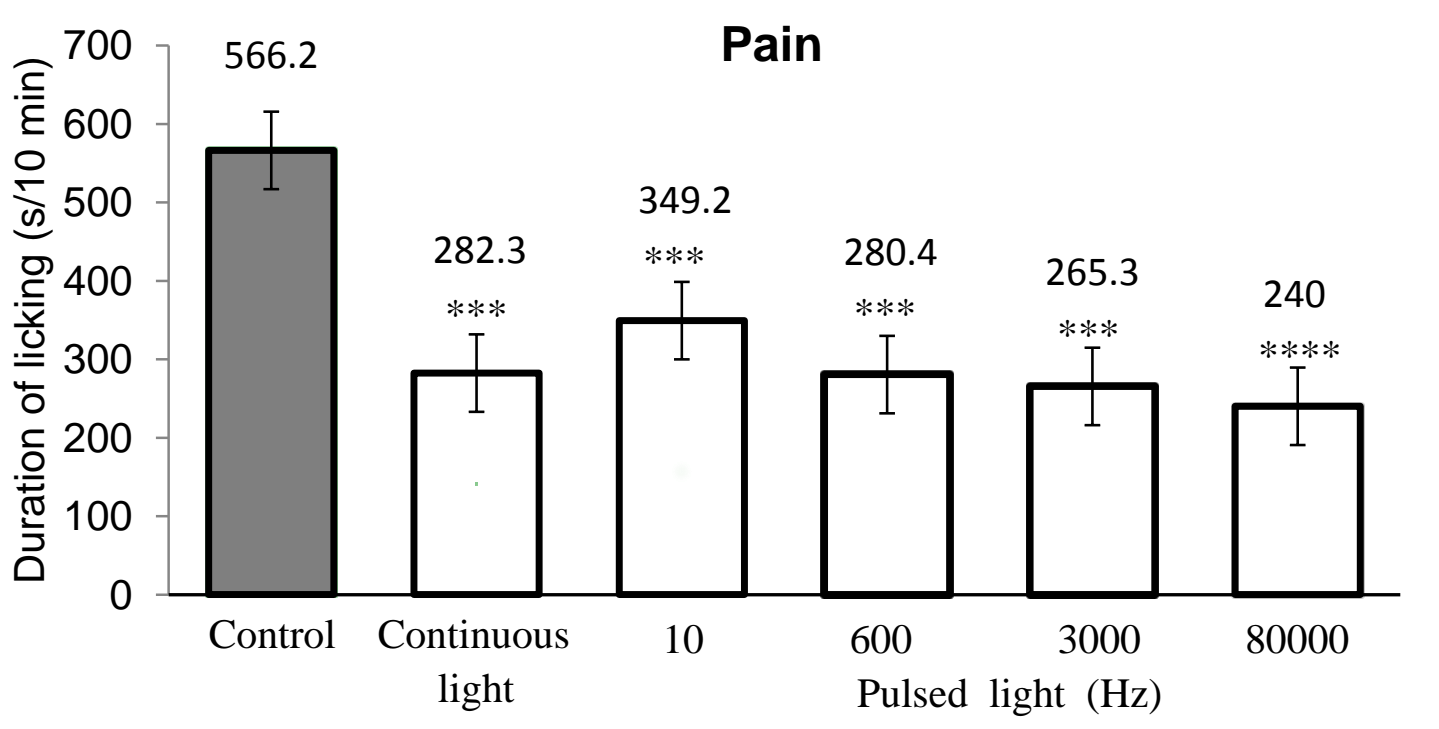

Fig. 4 Pain reaction after blue+IR light applications on the locus of pain, as compared with control data (without light). Bars represent mean \pm S.E.M. The numbers above the bars - the duration of the pain reaction within 60 minutes of observation in seconds.

Significant differences with the control: **** $P<0.001 ; * * * P<0.01$.

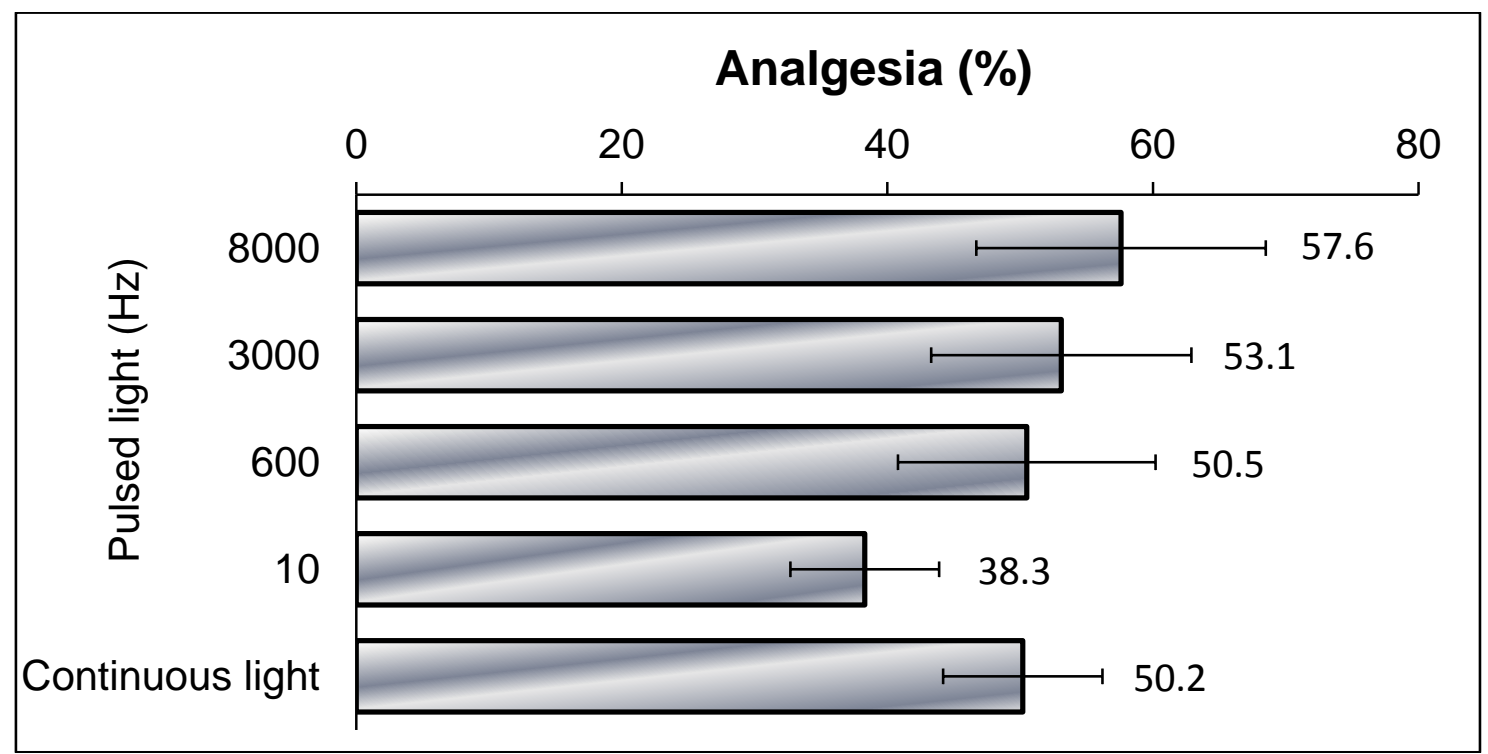

Fig. 5 Dependence of the analgesic effect produced by blue+IR of MEDOLIGHT-BluDoc device on the frequency of light pulsation.

The numbers next to the columns on the right-analgesia in $\%$.

Comparison of the effectiveness of analgesic action of different modes of blue+IR LED light is shown in Fig. 5. Light pulsing frequency of $8000 \mathrm{~Hz}$ most strongly weakened the pain. Analgesia made $57.6 \%$, that is, pain reaction weakened more than twice. The minimum efficacy was registered at light application with pulsing frequency $10 \mathrm{~Hz}$. Analgesia in this case was
$38.3 \%$. The difference between the two experimental groups $(10 \mathrm{~Hz}$ and $8000 \mathrm{~Hz}$ ) is statistically significant. Application of CL or PL with frequency of $600 \mathrm{~Hz}$ attenuated pain for $50.2 \%$ and $50.5 \%$ respectively. The difference between these groups is not significant.

Of the non-painful behavioral reactions, especially vivid were sleep changes. In Figs. 6 and 7, in all the 
experimental groups, the total sleep dynamics and its duration differed from that observed in the control group. A significant difference with the control is very high (Tables 1 and 2). PL more increased duration of sleep than CL. In groups where PL blue+IR was used, sleeping period increased at least 2.8 times (at a pulsing frequency of $600 \mathrm{~Hz}$ ) and maximum 3.8 times (at 10 $\mathrm{Hz}$ pulsing frequency). CL increased sleep duration 2.3 times. Thus, substantial growth of duration of dream specifies on ability of blue light with an infra-red component to provide not only analgetic but also sedative effect.

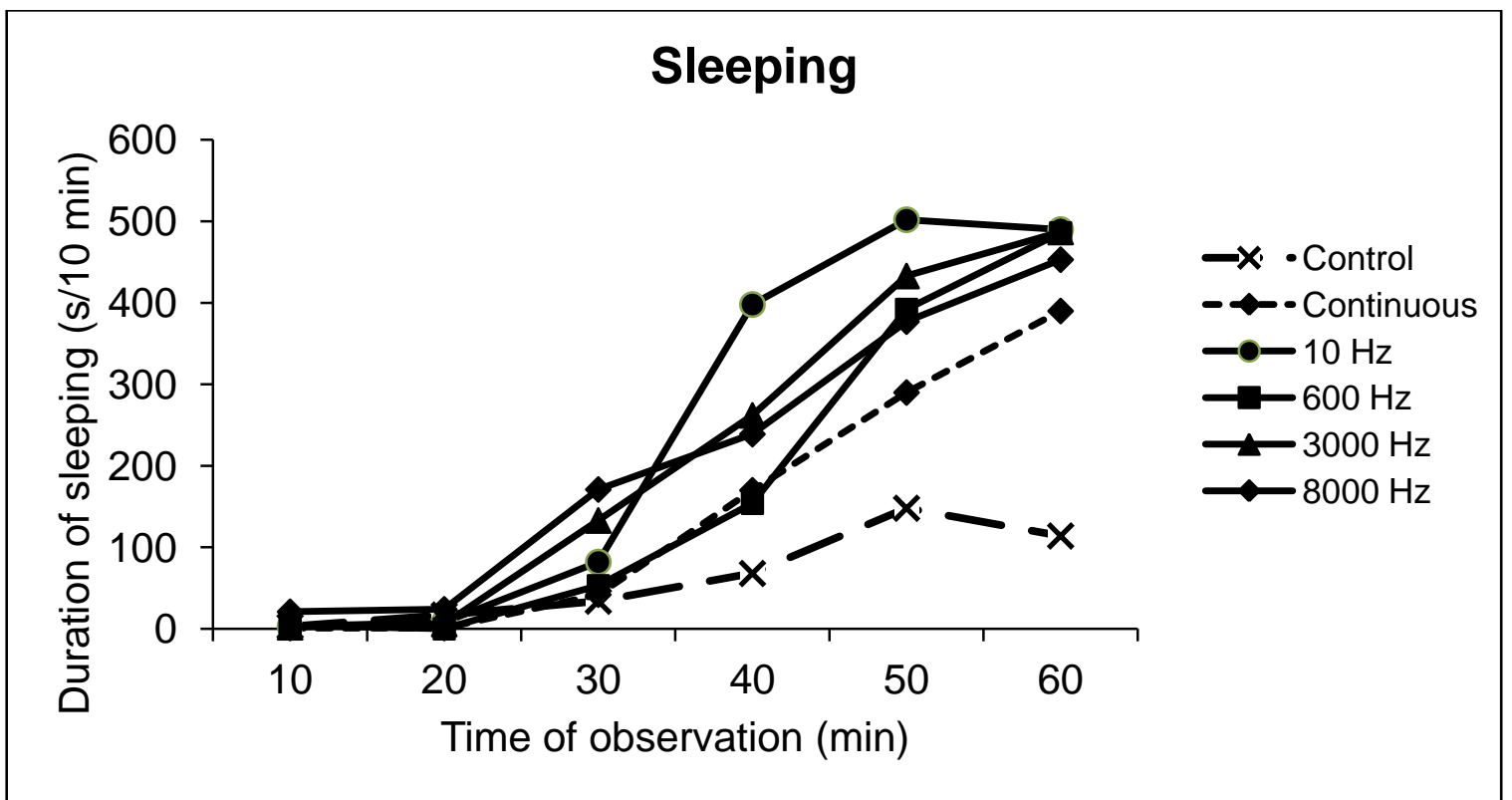

Fig. 6 Sleep dynamics in animals of five experimental groups who received blue+IR LED light influence on the pain locus, as compared with the control (mean values).

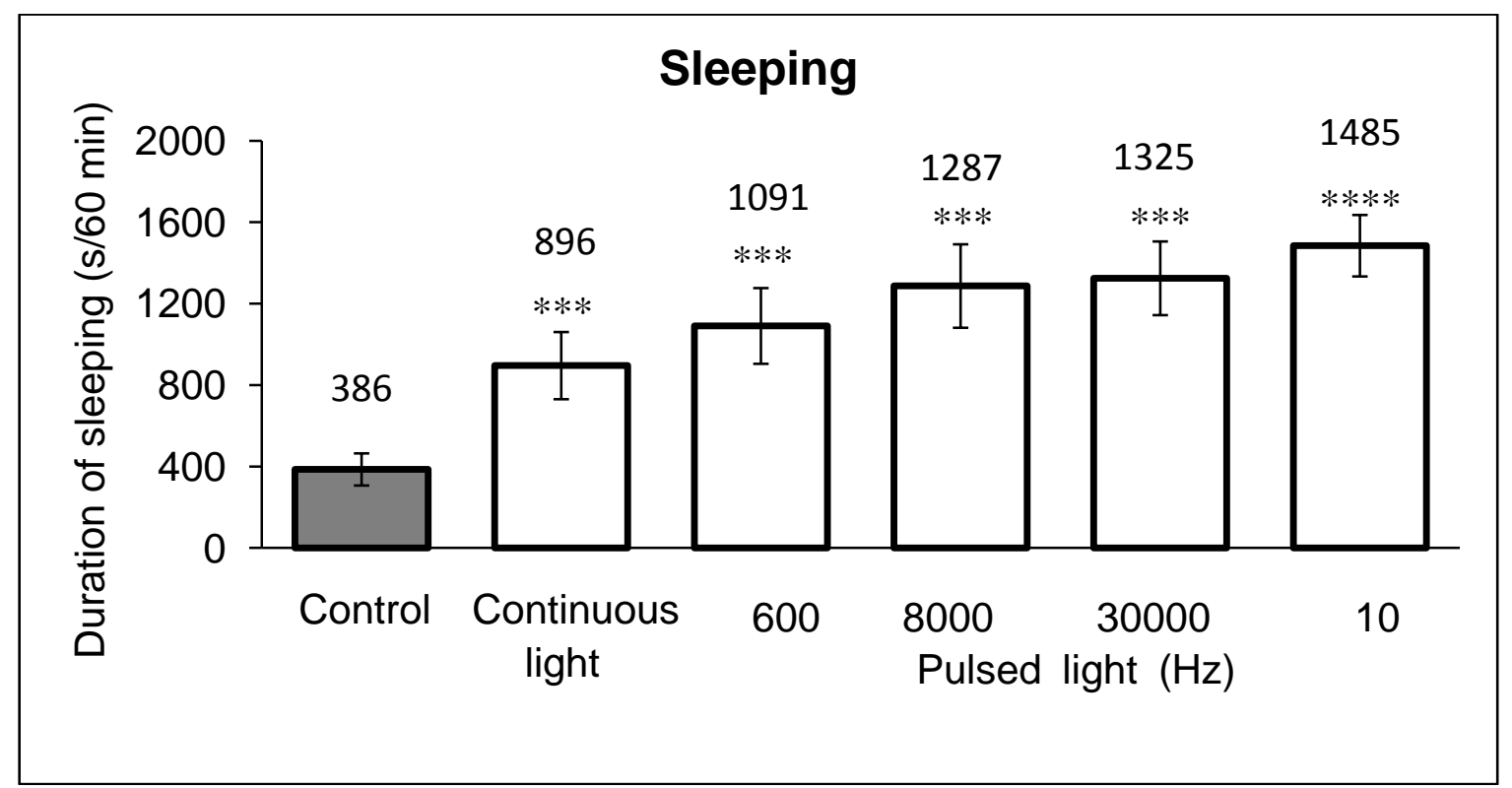

Fig. 7 Sleep duration in animals who received influence on pain locus by blue LED light+IR, as compared with the control. The numbers above the bars - the duration of sleep in seconds during 60 minutes of observation. 


\section{Formalin}

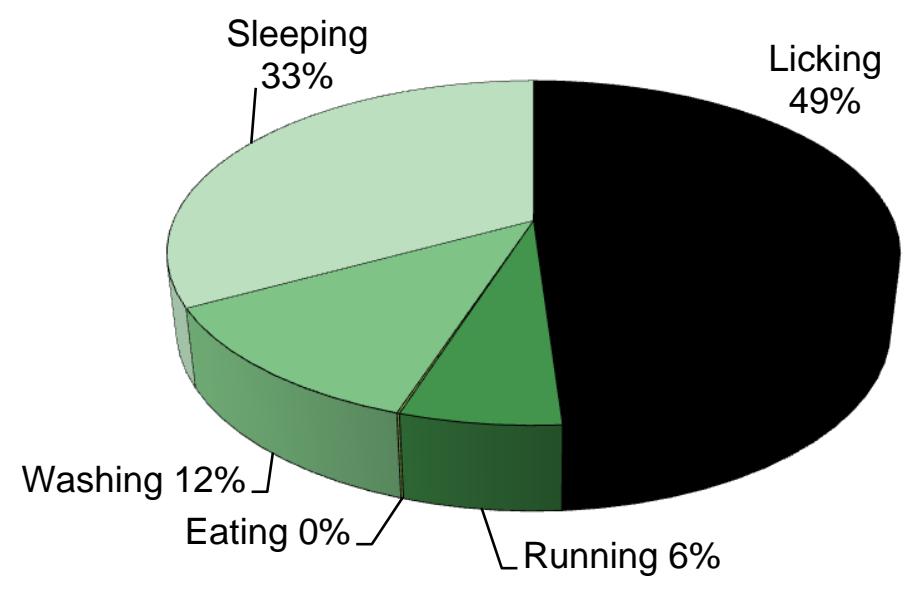

\section{Formalin + LED Liht $8000 \mathrm{~Hz}$}

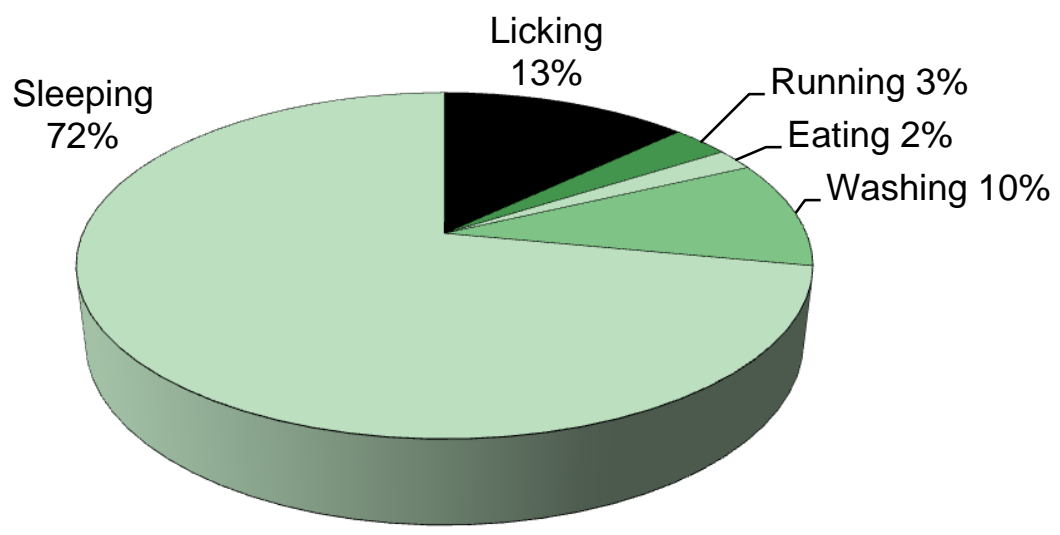

Fig. 8 Comparison of behavioral reactions of animals after formalin injection without exposure to light $(n=10)$ and after a 10-minute session on the locus of pain by blue+IR PL with pulsing frequency of $8000 \mathrm{~Hz}(n=10)$.

The amount of time spent on meals, of the observed animals increased about 30-times (Table 2). It also testifies to pain relief and sedative effect of this light.

Thus, in all the experiments, in which we used blue+IR LED light, there has been a reduction in the duration of pain response (licking the pain locus) and increase of non-painful reaction time (sleeping, eating, running, washing).

The intensity of pain can be described as a proportional ratio between the duration of painful and non-painful reactions. If to take the time spent for all the 5 registered reactions as $100 \%$ (full circle), and each reaction to assess in \% of the total time (Fig. 8), then pain relief, when exposed to light, is clearly noticeable. To illustrate, we have taken the group, with the maximum analgesic effect. You can see that after applying blue+IR PL pain behavioral response is only a small part $(13 \%)$ of the total time, and most $(72 \%)$ takes sleep. The group, which received imitation of light therapy session, the painful behavioral responses constituted the greatest percentage of time (49\%). Sleep in this group made up only $33 \%$.

Based on the studies mentioned above, we concluded that the blue LED light MEDOLIGHT-BluDoc device (continuous and pulsing modes) has significant analgesic and sedative effects. 


\section{Discussion}

The main results of this study are as follows: (1) It is proved that the LED blue and LED blue+IR light significantly relieve pain in animals with experimentally induced inflammatory locus (formalin test). (2) In addition to the analgesic effect, we observed sedative effect of blue light (with IR component and without it). (3) It is established that the PL blue $+\mathrm{IR}$ is more effective than CL. Of the four studied light pulsed frequencies $(10 \mathrm{~Hz}, 600 \mathrm{~Hz}, 3000$ $\mathrm{Hz}$ and $8000 \mathrm{~Hz}$ ), the greatest effect was recorded at a frequency of $8000 \mathrm{~Hz}$, and the lowest at $10 \mathrm{~Hz}$.

\subsection{Effects of CL Blue and Blue+IR}

We have previously demonstrated $[11,12]$ that blue halogen polarized light attenuates formalin-induced pain response in mice. Analgesia was $31.5 \%$. Blue LED light used in this work was more effective. In similar experimental conditions, blue CL suppressed pain for $44 \%$, and with the addition of IR fragment analgesia increased up to $50.1 \%$.

The role of low-intensity infrared radiation in different models of experimentally induced pain in animals is described in the literature in considerable details. It is shown the anti-nociceptive and anti-inflammatory effect of infrared light at a visceral pain, pain in the temporomandibular joint, formalin-induced inflammatory pain in extremity, pain in mice with closed craniocerebral trauma et al. [16-18]. However, the analgesic effect observed in our experiments using the blue and infrared LEDs (50.1\%), cannot be attributed solely to the IR irradiation. The use of the blue LEDs (without IR) provided close analgesic effect (44\%). The difference between the two experimental groups was not statistically significant. Thus, the blue light also has a marked antinociceptive effect. This is confirmed by numerous clinical studies.

The blue light is considered to be an effective factor in physiotherapy, having anti-inflammatory, sedative, relaxing effect mainly on the skin, mucous membranes, lungs, intestine, central nervous system and the blood of man. Blue light treatment is widely used at internal diseases, dermatology, ophthalmology, phthisiatry, at colds and other disorders.

Our experimental data suggest that blue LED light (as with IR or without an IR component) not only reduces pain, but also has a powerful sedative (relaxing) influence. This is evidenced by the increase of sleep and eating time duration. In the group where the blue light was used, the sleep period increased 2.4 times, while the animals treated with the session of blue+IR -2.3 times compared with the control.

\subsection{Effects of Pulsed Blue+IR LED Light}

In this article, we are the first who carried out comparison of the biological effectiveness of CL and PL blue +IR in the same experimental conditions. We obtained strong evidence of a more powerful analgesic action of PL. Of the four tested frequencies of PL (10 $\mathrm{Hz}, 600 \mathrm{~Hz}, 3000 \mathrm{~Hz}$ and $8000 \mathrm{~Hz}$ ), $8000 \mathrm{~Hz}$ frequency was the most effective. Analgesia, due to application of blue+IR PL with pulsing frequency $8000 \mathrm{~Hz}$ to the locus of inflammation was $57.6 \%$, which was significantly higher than that of a similar light with a continuous mode- $50.1 \%$. $10 \mathrm{~Hz}$ frequency was the least effective ( $38.3 \%$ analgesia).

Our results are consistent with the literature data, according to which effects of the PL (LASER or LED) differ from the effects of CL [19]. Thus, it was shown that the laser radiation with a wavelength of $670 \mathrm{~nm}$ in a pulsing mode $(10 \mathrm{~Hz}, 25 \mathrm{~Hz}$ and $50 \mathrm{~Hz})$ promotes wound healing better than the same laser in continuous mode [20]. Studies on mice with experimentally induced brain injury have shown that the laser $(810$ $\mathrm{nm}$ ), pulsed with a frequency of $10 \mathrm{~Hz}$, has a greater therapeutic effect than continuous. Efficacy was assessed by neurological parameters and histological results of traumatized mice [18]. In the model of formalin-induced pain, Susko et al. [21] found that use of pulsed light red+IR (particularly with a frequency of $10 \mathrm{~Hz}$ and $8000 \mathrm{~Hz}$ ) is more efficient than a continuous one. 
It is suggested that the high efficiency of the pulsing light due to the fact that it penetrates deeper into tissue than continuous light due to the increased penetration of photons at impulse peak [22].

Our studies have shown that non-painful behavioral responses are also stronger affected by the PL. Pulsed light prolonged sleep duration better than continuous. In groups where blue+IR LED light was used, sleep duration increased at least 2.8 times (at a frequency of $600 \mathrm{~Hz}$ ) and maximum-3.8 times (at a frequency of $10 \mathrm{~Hz}$ ). At the same time, CL increased sleep duration 2.3 times. Approximately 30 times in experimental animals increased the eating period. It also testifies to pain relief and sedative effect of the light.

Thus, when applied to the locus of inflammation the LED blue+IR PL or CL, it was found that the PL not only suppress the pain stronger, but also has a more powerful sedative effect.

\subsection{Mechanisms of Extra-ocular Action of Blue+IR Light}

Effects described in our study, were obtained by application of blue or blue+IR LED light on the locus of inflammation located on the foot of the hind limb of the mouse. A small area of skin was subjected to light irradiation. Visual analyzer did not participate in this process, because at the time of exposure each animal was in a special chamber. The question is how to explain the biological effects observed in our experiments.

Currently it considered proven that the light can be perceived not only by the retina, but also by the light-sensitive proteins contained in the skin of animals and humans. Skin - the largest organ of perception, which absorbs $80 \%$ of the light, and only $20 \%$ of the light impulses enter the brain via the optic nerve. Depending on the wavelength, light of different colors can penetrate $10 \mathrm{~mm}$ into the skin, affecting on various processes in the body.

The light-sensitive proteins that are able to absorb light quanta, are called chromophores. Red light receptors-phytochromes, receptors of blue light - cryptochromes. Any photo-biological process begins with the light energy absorbing by chromophore. The chromophore has a specific spectrum (band) of absorption. After absorbing light quantum chromophore passes from a stable state to an excited. The skin contains a lot of chromophores, some of which are photodynamically active, others passive [22].

The energy of the electromagnetic field and the light emission in the interaction with body tissue are converted into other forms of energy (chemical, thermal, etc.). This serves as a starting element of many physic-chemical and biological reactions that form the final therapeutic effect. In addition, each type of the electromagnetic field and radiation evokes unique, peculiar only to it photo biological processes. They determine the specificity of their therapeutic effects. The longer the wavelength, the deeper radiation penetrates.

Clinical observations suggest that blue light has anti-inflammatory, sedative and antibacterial properties. It is an effective treatment for inflammatory skin diseases, as it inactivates micro flora that caused acne.

Blue light penetrates relatively not deep through the skin $(0.5-1 \mathrm{~mm})$. However, it has the most effective wavelength for photo activation of endogenous porphyrins, as it has the largest ratio of the photo excitation. Porphyrins are widespread in nature pigments. Porphyrins excitation during absorption of light leads to formation of singlet oxygen and reactive radicals [23]. Recent researches have shown that blue light may increase nitric oxide production and related reactive oxygen species.

It is assumed that some of the therapeutic effects of blue light can be considered in connection with changes in metabolism of $\mathrm{L}$-arginine under the action of light (400 $\mathrm{nm}$ to $510 \mathrm{~nm})$. Enhancement of L-arginine in the plasma increases secretion of several hormones, including insulin, glucagon, growth 
hormone and adrenokateholamin [24]. L-arginine is a unique substrate for agmatine production, which has a great therapeutic potential for chronic pain and brain damage. In addition, it completely inhibits the activity of all isoforms of nitric oxide synthesis, protecting the body from negative effects of its excess [26]. This can explain some of the observed effects of the blue light: blood pressure lowering, analgesic effect, insulin secretion regulation, anti-inflammatory effect. A number of studies prove that the mechanism of blue light action is based on increasing the energy capacity of tissues at the expense of energy synthesis increase in cells mitochondria [25].

The infrared irradiation penetrates deeper into the skin. It has pronounced anti-inflammatory properties. It is known that infrared light causes local increase of the exposed skin temperature for to $1-2^{\circ} \mathrm{C}$ and evokes local reaction of surface vasculature. Vascular response reflected in increase of local blood flow and the volume of circulating blood in tissues. Activation of the microvasculature net and increased vascular permeability promotes dehydration of the inflammatory locus and removal of cellular debris, acceleration of wound healing.

However, besides heating there takes place the direct effect of photons on biological processes in tissues. It is shown that infrared laser radiation can effectively penetrate into biological tissue, including the central nervous system causing effects such as stimulation of nerve regeneration [26-28] and ATP synthesis increase [29]. These effects can be attributed to photochemical mechanisms, based on the light absorption by chromophores, but not heat of tissues [29-32].

In our case, a combination of blue light and infrared irradiation, due to the synergy of their action, effectively weakened the pain response to inflammation and provided a powerful relaxing (sedative) influence. These data suggest that the blue LED light (as with an infrared component, and without it) can be used in various pain syndromes, as well as under stress, insomnia, fatigue. Information about blue light ability to prevent stress development, are presented in the literature [33].

\section{Conclusion}

Our findings suggest that the blue and blue+infrared irradiation of MEDOLIGHT-BluDoc device significantly weakens the formalin-induced pain response. At equal application on the locus of inflammation, continuous blue light suppressed pain for $44 \%$, and CL blue+IR - for $50.1 \%$. The effect of PL blue+IR depended on the frequency of pulsation. Maximum analgesia was registered at frequency of $8000 \mathrm{~Hz}(57.6 \%)$, and the minimum-at $10 \mathrm{~Hz}$ $(38.3 \%)$. It was found that both the blue and blue+IR LED light at all investigated regimes have a significant sedative effect along with the analgesic one. We can assume that these results can be the basis for application of the studied variants of blue light for the therapeutic purposes. In particular, we can talk about its prospects, not only for pain relief, but also at stress, insomnia and fatigue [34].

\section{References}

[1] Karandashov, V. I., Petuchov, E. B., and Srodnykov, V. C. 2001. Phototherapy. Moskow, Medicina. (in Russian)

[2] Vekshyn, N. L. 1991. "Light-dependent Phosphorylation in the Mitochondria." Molec. Biol. 25 (1): 54-9. (in Russian)

[3] Danylov, A. D., Karandashov, V. I., and Slesarev, V. I. 2011. "Phototherapy by Irradiators Blue Spectrum in Terms of the Physics and Chemistry of Water-Based Systems." Non-Drug Medicine 10 (2): 24-5. (in Russian)

[4] Gulyar, S. A., ed. 2009. Anthology of Light Therapy. Medical Bioptron Technologies. Kyiv: Bogomoletz Institute of Physiology at the National Academy of Sciences of Ukraine. (in Russian)

[5] Gulyar, S. A., and Kosakovskyi, A. L., eds. 2011. Bioptron-PILER-light Application in Medicine. 2nd ed. Kyiv: Bogomoletz Institute of Physiology at the National Academy of Sciences of Ukraine. (in Russian)

[6] Gulyar, S. A., Limansky, Yu. P., and Tamarova, Z. A. 2007. Pain Color-therapy: Treatment of Pain Syndromes by Polarized Color Light. 2nd ed. Kyiv: Bogomoletz Institute of Physiology at the National Academy of Sciences of Ukraine. (in Russian) 
[7] Moskvyn, C. V., and Bujlyn, V. A., eds. 2000. Low-intensity Laser Therapy. Moskow: Techniks. (in Russian)

[8] Ponomarenko, G. N., and Vorob'ev, M. G. 2005. Manual of Physiotherapy. St. Petersburg: Baltic. (in Russian)

[9] Gulyar, S., and Tamarova, Z. 2015. "Antipain Effect of the Red-infrared Polarized LED Irradiation ECOZEPT Device." In Proc. XLIII Intern. Sci-Pract. Conf.: Application of Lasers in Medicine and Biology. Kharkiv: 86-9. (in Russian)

[10] Gulyar, S., and Tamarova, Z. 2017. "Analgesic Effects of the Polarized Red+Infrared LED Light." J. of US-China Medical Science 14 (2): 47-57.

[11] Tamarova, Z., Limansky, Y., and Gulyar, S. 2009. "Antinociceptive Effects of Color Polarized Light in Animal with Formalin Test." Fiziol. J. 55 (3): 81-93. (in Ukrainian)

[12] Limansky, Y., Gulyar, S., and Tamarova, Z. 2009. "BIOPTRON-analgesia: 12. Role of Color in Tonic Pain Suppression." Anthology of Light Therapy. Medical BIOPTRON Technologies. Kyiv: Bogomoletz Institute of Physiology at the National Academy of Sciences of Ukraine: 722-31. (in Russian)

[13] Dubuisson, D., and Dennis, S. 1977. "The Formalin Test: A Quantitative Study of the Analgesic Effects of Morphine, Nepedioine and Brain Stem Stimulation in Rats and Cats." Pain 4 (2): 161-74.

[14] Hunskaar, S., and Hole, K. 1987. "The Formalin Test in Mice: Dissociation Between Inflammatory and non-Inflammatory." Pain 30 (1): 103-4.

[15] Kavaliers, M., and Hirst, M. 1983. "Daily Rhythms of Analgesia in Mace: Effects of Age and Photoperiod." Brain Res. 279 (1-2): 387-93.

[16] Erthal, V., Silva, M. D., Cidral-Filho, F. J., Santos, A. R., and Nohama, P. 2013. "ST36 Laser Acupuncture Reduces Pain-Related Behaviour in Rats: Involvement of the Opioidergic and Serotonergic Systems." Lasers Med. Sci. 28 (5): 1345-51.

[17] Barrettoa, S. R., de Meloa, G. C., dos Santos, J. C., de Oliveira, M. G., Pereira-Filhoa, R. N., Alves, A. V., Ribeiro, M. A., Lima-Verde, I. B., Quintans Júnior, L. J., de Albuquerque-Júnior, R. L., and Bonjardim L. R. 2013. "Evaluation of anti-Nociceptive and anti-Inflammatory Activity of Low-level Laser Therapy on Temporomandibular Joint Inflammation in Rodents." J. of Photochemistry and Photobiology B: Biology 129 (5): 135-42.

[18] Takahiro, A., Weijun, X., Tao, X., Tianhong, D., and Sulbha, K. S. 2011. "Comparison of Therapeutic Effects between Pulsed and Continuous Wave $810 \mathrm{~nm}$ Wavelength Laser Irradiation for Traumatic Brain Injury in Mice." PLoS One 6 (10): e26212.
[19] Hashmi, J. T., Huang, Y. Y., Sharma, S. K., Kurup, D. B., De Taboada, L., Carroll, J. D., and Hamblin, M. R. 2010. "Effect of Pulsing in Low-level Light Therapy." Lasers Surg. Med. 42 (6): 450-66.

[20] Kymplova, J., Navratil, L., and Knizek, J. 2003. "Contribution of Phototherapy to the Treatment of Episiotomies.” J. Clin. Laser Med. Surg. 21 (1): 35-9.

[21] Sushko, B., Lymansky, Yu., and Gulyar, S. 2007. "Action of the Red and Infrared Electromagnetic Waves of Light-emitting Diodes on the Behavioural Manifestation of Somatic Pain." Fiziol. Zh. 53 (3): 51-60.

[22] Konig, K., Ruck, A., and Schneckenburger, H. 1992. "Fluorescence Detection and Photodynamic Activity of Endogenous Protoporphyrin in Human Skin.” Opt. Eng. 31: 1470-4.

[23] Manyak, M. J. 1990. "Photodynamic Therapy: Present Concepts and Future Applications." Cancer. J. 3: 104-9.

[24] Makela, A. M. 2005. "Role of L-arginine in the Biological Effects of Blue Light." Proc. SPIE 5968, Laser Florence 2004: A Window on the Laser Medicine World, 596805. Accessed October 25, 2005. doi:10.1117/12.660038.

[25] Karandashov, V. I. 2013. "Biological Effects of Blue Light Irradiation and Perspectives of Its Application in Practical Medicine." Photobiological and Experimental Photomedicine 1, 2: 98-106. (in Ukrainian)

[26] Anders, J. J., Borke, R. C., Woolery, S. K., and van de Merwe, W. P. 1993. "Low Power Laser Irradiation Alters the Rate of Regeneration of the Rat Facial Nerve." Lasers Surg. Med. 13 (1): 72-82.

[27] Anders, J. J., Geuna, S., and Rochkind, S. 2004. "Phototherapy Promotes Regeneration and Functional Recovery of Injured Peripheral Nerve." Neurol. Res. 26 (2): 233-9.

[28] Xingjia, W., Anton, E. D., Mario, J. C., Angela, G. V., Rosemary, C. B., Jackson, S., and Juanita, J. A. 2009. "810 nm Wavelength Light: An Effective Therapy for Transected or Contused Rat Spinal Cord." Lasers Surg. Med. 41 (1): 36-41.

[29] Mochizuki-Oda, N., Kataoka, Y., Cui, Y., Yamada, H., Heya, M., and Awazu, K. 2002. "Effects of Near-infra-red Laser Irradiation on Adenosine Triphosphate and Adenosine Diphosphate Contents of Rat Brain Tissue." Neurosci. Lett. 323 (3): 207-10.

[30] Byrnes, K. R., Waynant, R. W., Ilev, I. K., Wu, X., Barna, L., Smith, K., Heckert, R., Gerst, H., and Anders, J. J. 2005. "Light Promotes Regeneration and Functional Recovery and Alters the Immune Response after Spinal Cord Injury." Lasers Surg. Med. 36 (3): 171-85.

[31] Karu, T. 1999. "Primary and Secondary Mechanisms of Action of Visible to Near-IR Radiation on Cells." $J$. Photochem. Photobiol. B. 49 (1): 1-17. 
[32] Castro-e-Silva, O., Zucoloto, S., Marcassa, L. G., Marcassa, J., Kurachi, C., Ramalho, F. S., Ramalho, L. N., and Bagnato, V. S. 2003. "Spectral Response for Laser Enhancement in Hepatic Regeneration for Hepatectomized Rats.” Lasers Surg. Med. 32 (1): 50-3.
[33] Volpato, J., and Barreto, K. 2001. "Environmental Blue Light Prevents Stress in the Fish Nile Tilapia." Braz. J. Med. Biol. Res. 34 (8): 1041-5.

[34] Gulyar, S. A. 2016. Medolight: Basic Healing Effects of LED Device. 5th ed., expand. Kyiv, IMIC, 1-64. 